\title{
A NOTE ON GENERALIZED TAUBERIAN THEOREMS
}

\author{
C. T. RAJAGOPAL
}

1. Introduction. Suppose that $A(u)$ is a function of bounded variation in every finite interval of $u \geqq 0, A(0)=0,{ }^{1}$ and that the $\Phi$-transform of $A(u)$, namely,

$$
\Phi(t)=\int_{0}^{\infty} \phi(u t) d\{A(u)\}
$$

is convergent for $t>0$, the function $\phi(u)$ satisfying the following conditions.

$\mathrm{C}(\mathrm{i})$ For $u \geqq 0, \phi(u)$ is positive, continuous, and monotonic decreasing;

(ii) $\phi(0)=1, \int^{\infty}(\phi(u) / u) d u$ is convergent;

(iii) for $u \geqq 0, \phi(u)$ has a continuous derivative $-\psi(u)$ which is, on account of (i), negative and such that

$$
\phi(u)=\int_{u}^{\infty} \psi(x) d x ;^{2}
$$

(iv) for $u \geqq 0, \psi(u)$ is monotonic decreasing and has a continuous derivative.

Then a generalized Tauberian theorem in the sense in which the expression is used in the present context is a "converse" theorem which enables us to relate the behaviour of $A(u)$ as $u \rightarrow \infty$ to that of $\Phi(t)$ as $t \rightarrow+0$, under a suitable Tauberian condition. In the usual terminology, such a theorem may be called an " $O$-inversionssatz" when it has a Tauberian $o_{L}$ - or $o_{R}$-hypothesis and a conclusion which relates lim sup (or inf) $A(u), u \rightarrow \infty$, to lim sup (or inf) $\Phi(t), t \rightarrow+o$; an " $o$-inversionssatz," when it assumes a Tauberian $O_{L}$ - or $O_{R}$-condition and deduces the convergence of $A(u)$ as $u \rightarrow \infty$ from that of $\Phi(t)$ as $t \rightarrow+0$.

It is the object of this note to show that the classical inversion theorems of Karamata and Ramaswami, of the two kinds mentioned

Received by the editors April 10, 1950. ized.

${ }^{1}$ The condition $A(0)=0$ is included in the usual assumption that $A(u)$ is normal-

2 The monotony of $\phi$ and the convergence of the integral in (ii) ensure that $\phi(u) \rightarrow 0$ as $u \rightarrow \infty$. Hence $\int_{5}^{\infty} \psi(u) d u$ exists, $\phi(u)=\int_{u}^{\infty} \psi(x) d x+$ a constant, where the constant is seen to be 0 by making $u \rightarrow \infty$.

It may be noticed that the usual assumption, in addition to $\mathrm{C}$ (ii), of the existence of $\int_{0}[1-\phi(u)] / u d u$ is implied in the fact $\lim _{u \rightarrow 0}[1-\phi(u)] / u=\lim _{u \rightarrow 0}-\phi^{\prime}(u)$ $=\lim _{u \rightarrow 0} \psi(u)$. 
above, which appear as corollaries in $\$ 4$, can be treated uniformly by means of two principal results, Theorems A and B of $\$ 3$, embodying a technique of Szász [6, 7]. ${ }^{3}$

2. Lemmas. There are some results about the $\Phi$-transform which are needed to establish our main theorems. These results are either known or can be obtained by known methods and are collected below.

Lемма 1. (i) Suppose that, in the interval $u \geqq 0, \phi(u)$ is positive, differentiable with continuous derivative, ultimately monotonic, and tending to 0.

(ii) Suppose that $A(u)$ is a function of bounded variation in every finite interval of $u \geqq 0, A(0)=0$. Then, if

$$
\Phi(t)=\int_{0}^{\infty} \phi(u t) d\{A(u)\} \text { converges for } t=t_{0}>0,
$$

it follows that

$$
\Phi\left(t_{0}\right)=t_{0} \int_{0}^{\infty} \psi\left(u t_{0}\right) A(u) d u, \quad \psi(u) \equiv-\phi^{\prime}(u)
$$

This result is proved elsewhere [4, Lemma 2] as for the particular case of the Laplace transform where $\phi(u)=e^{-u}[8$, p. 41, Theorem $2.3 a]$.

Lemma 2. Suppose that $\phi(u)$ satisfies conditions $\mathrm{C}(\mathrm{i})-(\mathrm{iv})$ of $\$ 1$ and $A(u)$ is as in (ii) of Lemma 1. If

$$
\Phi(t)=\int_{0}^{\infty} \phi(u t) d\{A(u)\} \text { converges for } t>0,
$$

then the integral

$$
\Phi_{1}(t)=t \int_{0}^{\infty} \psi(u t) \frac{A_{1}(u)}{u} d u, \quad \text { where } A_{1}(u) \equiv \int_{0}^{u} A(x) d x,
$$

converges for $t>0$, and

$$
\begin{aligned}
\liminf _{u \rightarrow \infty} \frac{A_{1}(u)}{u} & \leqq \liminf _{t \rightarrow+0} \Phi(t) \leqq \lim _{t \rightarrow+0} \sup \Phi_{1}(t) \leqq \limsup _{t \rightarrow+0} \Phi(t) \\
& \leqq \limsup _{u \rightarrow \infty} \frac{A_{1}(u)}{u} .
\end{aligned}
$$

These inequalities, with the omission of the extreme members,

${ }^{3}$ Numbers in brackets refer to the references at the end of the paper. 
have been proved by Minakshisundaram [2, \$2] just as for a similar result of Szász in the case $\phi(u)=e^{-u}[7$, Lemma 1]. The inequalities, with the omission of the middle members, can also be proved as for their well known special case $\phi(u)=e^{-u}$.

Lемма 3. Suppose that, for $u \geqq 0, \psi(u)$ is positive, differentiable with continuous derivative, ultimately monotonic, and

$$
\int_{0}^{\infty} \psi(u) d u=1, \quad \int_{0}^{\infty} e^{i x} \psi(u) d u \neq 0 \quad(-\infty<x<\infty) .
$$

Then, if $p(u) \geqq 0$ for $u \geqq 0$,

$$
t \int_{0}^{\infty} \psi(u t) p(u) d u, \quad t>0, \quad \text { converges as } t \rightarrow+0,
$$

we have

$$
\lim _{x \rightarrow \infty} \frac{1}{x} \int_{0}^{x} p(u) d u=\lim _{t \rightarrow+0} t \int_{0}^{\infty} \psi(u t) p(u) d u .
$$

This lemma is a generalization for the $\Phi$-transform of Doetsch's "positive" Tauberian theorem. It may be established as shown elsewhere [4, Theorem 1], by means of Pitt's "one-sided" modification of Wiener's general Tauberian theorem [8, pp. 220-221, Theorem $13 b]$ and the next lemma.

Lemma 4. Suppose that for $u \geqq 0, \psi(u)$ is positive, differentiable, ultimately monotonic. Then the hypotheses, $p(u) \geqq 0$ for $u \geqq 0, \int_{0}^{\infty} \psi(u) d u$ exists,

$$
t \int_{0}^{\infty} \psi(u t) p(u) d u \text { exists for } t>0
$$

ensure the conclusion

$$
\begin{aligned}
\underset{x \rightarrow \infty}{\limsup } \frac{1}{x} \int_{0}^{x} p(u) d u \leqq C \limsup _{t \rightarrow+0} t \int_{0}^{\infty} \psi(u t) p(u) d u, \\
C=\text { a constant depending on } \psi \text { only. }
\end{aligned}
$$

This result, due to Minakshisundaram [2, Theorem 1], generalizes a well known theorem of Hardy and Littlewood.

Lemma 5. Let $\phi(u)$ satisfy the conditions $\mathrm{C}(\mathrm{i})$, (ii), (iii) of $\$ 1$. Let

$$
S(u)=\int_{0}^{u} s(x) d x, \quad F(t)=\int_{0}^{\infty} \phi(u t) s(u) d u .
$$


Then the hypothesis

$$
0 \leqq \lim _{u \rightarrow \infty} \sup u s(u)<\infty
$$

implies

$$
\underset{u \rightarrow \infty}{\operatorname{osc}} S(u)=\underset{t \rightarrow+0}{\operatorname{osc}} F(t)
$$

In particular, the hypothesis $\lim _{u \rightarrow \infty} u s(u)=0$ involves

$$
\lim _{u \rightarrow \infty} S(u)=\lim _{t \rightarrow+0} F(t)
$$

whenever the limit on the right side exists.

This result is also due to Minakshisundaram [2, \$3.3]. Its particular case is the 0 -Tauberian theorem in the usual form, for the $\Phi$ transform. The general case, as we shall see later, admits of a modification which dispenses with the hypothesis $\lim \sup u s(u)<\infty$.

LEMMA 6. If $b(u) \rightarrow 0$ as $u \rightarrow \infty$ and $\psi(u)$ is any function integrable in $(0, \infty)$, then $t \int_{0}^{\infty} b(u) \psi(u t) d u$ exists for $t>0$ and tends to 0 as $t \rightarrow+0$.

The proof of this lemma is obvious and omitted.

In the passage from our main theorems to the results of Ramaswami and Karamata referred to in $\$ 1$, we require the next three lemmas.

\section{LEMMA 7. If}

$$
\underset{u<u^{\prime}<\lambda u}{\text { lower bound }}\left\{A\left(u^{\prime}\right)-A(u)\right\} \geqq-w(\lambda) \text { for every } u>0, \lambda>1 \text {, }
$$

then

$$
A(u)-\frac{1}{u} \int_{0}^{u} A(x) d x>-w(\lambda) \frac{\lambda}{\lambda-1}, \quad u>0 .
$$

This is a result of Szász [6, Hilfssatz $1,(14)$, case $\beta=0]$.

LEMMA 8. If

$\underset{u \rightarrow \infty}{\lim \inf } \underset{u<u^{\prime}<\lambda u}{\text { lower bound }}\left\{A\left(u^{\prime}\right)-A(u)\right\} \geqq-w(\lambda) \rightarrow 0$ as $1<\lambda \rightarrow 1$,

then

$$
\lim _{u \rightarrow \infty} A(u)=\lim _{u \rightarrow \infty} \frac{1}{u} \int_{0}^{u} A(x) d x
$$

whenever the limit on the right side exists. 
This lemma is also given by Szász $[6$, Hilfssatz 3 , case $\beta=0]$.

\section{LEMMA 9. If}

$\underset{u \rightarrow \infty}{\lim \inf } \underset{u<u^{\prime}<\lambda_{u}}{\text { lower bound }}\left\{A\left(u^{\prime}\right)-A(u)\right\} \geqq-w(\lambda)=o_{L}(\lambda-1)$

$$
\text { as } 1<\lambda \rightarrow 1,
$$

then

$$
\underset{u \rightarrow \infty}{\operatorname{osc}} A(u)=\operatorname{osc}_{u \rightarrow \infty} \frac{1}{u} \int_{0}^{u} A(x) d x \text {. }
$$

Proof. We have, from Lemma 7,

$$
A(u)-\frac{1}{u} \int_{0}^{u} A(x) d x=o_{L}(1), \quad u \rightarrow \infty .
$$

Therefore

$$
\liminf _{u \rightarrow \infty} A(u) \geqq \liminf _{u \rightarrow \infty} \frac{1}{u} \int_{0}^{u} A(x) d x
$$

But since, in any case,

$$
\liminf _{u \rightarrow \infty} A(u) \leqq \liminf _{u \rightarrow \infty} \frac{1}{u} \int_{0}^{u} A(x) d x,
$$

we get at once

$$
\liminf _{u \rightarrow \infty} A(u)=\liminf _{u \rightarrow \infty} \frac{1}{u} \int_{0}^{u} A(\dot{x}) d x .
$$

The proof is now completed by establishing the equality of the corresponding upper limits, employing for the purpose an argument of Karamata which I have discussed elsewhere [for example, 3, proof of Theorem 1'].

\section{Main theorems.}

Theorem A. Let $\phi(u)$ satisfy the conditions C(i)-(iv) of $\$ 1$ and let us write $\psi(u)=-\phi^{\prime}(u)$ as there. Let $v(u)$ and its integral $V(u)=\int_{0}^{u} v(x) d x$ be such that

$$
v(u)=o_{L}(1), \quad u \rightarrow \infty,
$$

(3.2) $t \int_{0}^{\infty} \psi(u t) \frac{V(u)}{u} d u$ is convergent for $t>0$ and bounded as $t \rightarrow+0$. 
Then

$$
\operatorname{osc}_{x \rightarrow \infty} \int_{0}^{x} \frac{V(u)}{u^{2}} d u=\underset{t \rightarrow+0}{\operatorname{osc}} \int_{0}^{\infty} \phi(u t) \frac{V(u)}{u^{2}} d u,
$$

the integral on the right being convergent for $t>0$ as a result of our hypotheses. ${ }^{4}$

Proof. By virtue of (3.1)

$$
\frac{V(u)}{u}=o_{L}(1)
$$

$$
u \rightarrow \infty
$$

and so there is a constant $K>0$ such that

$$
\frac{V(u)}{u} \geqq-K, \quad u \geqq 0 .
$$

(3.5), in conjunction with (3.2), shows that we may choose $p(u)$ $=u^{-1} V(u)+k$ in Lemma 4 and conclude that

$$
\limsup _{x \rightarrow \infty} \frac{1}{x} \int_{0}^{x} \frac{V(u)}{u} d u<\infty .
$$

Hence, integrating by parts, we have

$$
\frac{V_{1}(x)}{x^{2}}+\frac{1}{x} \int_{0}^{x} \frac{V_{1}(u)}{u^{2}} d u=O_{R}(1), \quad x \rightarrow \infty
$$

$$
V_{1}(x) \equiv \int_{0}^{x} V(u) d u
$$

Now (3.4) and (3.6) give successively

$$
\begin{array}{cc}
\frac{V_{1}(x)}{x^{2}}=o_{L}(1), & x \rightarrow \infty, \\
\frac{V_{1}(x)}{x^{2}}=O_{R}(1)-\frac{1}{x} \int_{0}^{x} \frac{V_{1}(u)}{u^{2}} d u=O_{R}(1), & x \rightarrow \infty,
\end{array}
$$

and (3.7), (3.8) together give

$$
\frac{V_{1}(u)}{u^{2}}=O(1), \quad u \rightarrow \infty
$$

4 To avoid useless complications it may be supposed that, in this theorem as well as in the next, $V(u) / u^{2}=O(1), u \rightarrow+0$. 
Further, $V_{1}(2 u)-V_{1}(u)=\int_{0}^{u} V(2 u-x) d x$, which gives, after an integration by parts,

$$
V_{1}(2 u)-V_{1}(u)=u V(u)+\int_{0}^{u} x v(2 u-x) d x .
$$

Using (3.1) and (3.9) in (3.10) we find that $O\left(u^{2}\right)=u V(u)+o_{L}\left(u^{2}\right)$, $u \rightarrow \infty$, and therefore

$$
\frac{V(u)}{u}=O_{R}(1), \quad u \rightarrow \infty .
$$

(3.11) along with (3.4) leads to

$$
0 \leqq \lim _{u \rightarrow \infty} \sup \frac{V(u)}{u}<\infty,
$$

and this makes the integral on the right side of (3.3) convergent for $t>0$ as we can see by recalling the condition $C$ (ii) of $\$ 1$. It is further plain from (3.12) that we can take $s(u)=u^{-2} V(u)$ in Lemma 5 and reach the conclusion (3.3).

Lemma 1 shows that the conclusion (3.3) can also be written

$$
\operatorname{osc}_{x \rightarrow \infty} \int_{0}^{x} \frac{V(u)}{u^{2}} d u=\operatorname{osc}_{t \rightarrow+0} t \int_{0}^{\infty} \psi(u t) d u \int_{0}^{u} \frac{V(x)}{x^{2}} d x
$$

THEOREM B. Let $\phi(u)$ satisfy the conditions C (i)-(iv) of $\$ 1$ and further let $\psi(u)=-\phi^{\prime}(u)$ satisfy:

$$
\int_{0}^{\infty} u^{i x} \psi(u) d u \neq 0 \text { for any real } x \text {. }
$$

Suppose that

$$
V(u)=O_{L}(u), \quad u \rightarrow \infty,
$$

(3.16) $t \int_{0}^{\infty} \psi(u t) \frac{V(u)}{u} d u$ is convergent for $t>0$ and tends to 0 as $t \rightarrow+0$.

Then

$$
\begin{aligned}
& t \int_{0}^{\infty} \psi(u t) d u \int_{0}^{u} \frac{V(x)}{x^{2}} d x \text { is convergent for } t>0 \\
& \lim _{x \rightarrow \infty} \int_{0}^{x} \frac{V(u)}{u^{2}} d u=\lim _{t \rightarrow+0} t \int_{0}^{\infty} \psi(u t) d u \int_{0}^{u} \frac{V(x)}{x^{2}} d x
\end{aligned}
$$

whenever the limit on the right exists. 
Proof. In consequence of (3.15) there is a constant $K>0$ such that $V(u) / u+K \geqq 0$, and, in consequence of (3.16),

$$
t \int_{0}^{\infty} \psi(u t)\left[\frac{V(u)}{u}+K\right] d u \rightarrow K, \quad t \rightarrow+0
$$

Hence, using Lemma 3 , with $p(u)=u^{-1} V(u)+K$, we obtain

$$
\frac{1}{x} \int_{0}^{x}\left[\frac{V(u)}{u}+K\right] d u \rightarrow K, \quad x \rightarrow \infty,
$$

or

$$
\frac{1}{x} \int_{0}^{x} \frac{V(u)}{u} d u \rightarrow 0, \quad x \rightarrow \infty .
$$

An integration by parts gives

$$
\begin{aligned}
V_{1}(x) \equiv \int_{0}^{x} V(u) d u & =x \int_{0}^{x} \frac{V(u)}{u} d u-\int_{0}^{x} d u \int_{0}^{u} \frac{V(\tau)}{\tau} d \tau \\
& =x \int_{0}^{x} \cdots-\int_{0}^{\Delta} d u \int_{0}^{u} \cdots-\int_{\Delta}^{x} d u \int_{0}^{u} \cdots
\end{aligned}
$$

whence, choosing $\Delta$ suitably large and using (3.19), we get

$$
V_{1}(x)=o\left(x^{2}\right)+O(1)+o\left(x^{2}\right)=o\left(x^{2}\right), \quad x \rightarrow \infty .
$$

By an integration by parts we also get

$$
\int_{0}^{x} \frac{V(u)}{u^{2}} d u=\frac{V_{1}(x)}{x^{2}}+2 \int_{0}^{x} \frac{V_{1}(u)}{u^{3}} d u
$$

from which it follows that

$$
\begin{aligned}
t \int_{0}^{\infty} \psi(u t) d u \int_{0}^{u} & \frac{V(x)}{x^{2}} d x \\
& =t \int_{0}^{\infty}\left[\frac{V_{1}(u)}{u^{2}}+2 \int_{0}^{u} \frac{V_{1}(\tau)}{\tau^{3}} d \tau\right] \psi(u t) d u \\
& =I_{1}+I_{2}(\text { say })^{5}
\end{aligned}
$$

- Here we tacitly assume the convergence of $I_{1}, I_{2}$ for $t>0$ and therefore the truth of (3.17). To justify this assumption we note that (i) $I_{1}$ is convergent for $t>0$ as a result of (3.20) and the convergence of $\int^{\infty} \psi(u) d u$; (ii) the integral in (3.22) is convergent for $t>0$ as a result of $(3.20)$ and the convergence of $\int^{\infty}(\phi(u) / u) d u$, so that the second term on the right side of (3.21) which is the same as the integral in (3.22), by Lemma 1 , is also convergent for $t>0$. 
where, by Lemma 1,

$$
I_{2}=2 \int_{0}^{\infty} \phi(u t) \frac{V_{1}(u)}{u^{8}} d u
$$

From (3.20) we see that we can choose $b(u)=u^{-2} V_{1}(u)$ in Lemma 6, reaching the conclusion $\lim _{t \rightarrow+0} I_{1}=0$. This, in conjunction with (3.21), makes

$$
\lim _{t \rightarrow+0} I_{2}=\lim _{t \rightarrow+0} t \int_{0}^{\infty} \psi(u t) d u \int_{0}^{u} \frac{V(x)}{x^{2}} d x
$$

which we have assumed to exist. Again (3.20) enables us to choose $s(u)=u^{-3} V_{1}(u)$ in Lemma 5 and obtain from (3.22)

$$
\lim _{x \rightarrow \infty} 2 \int_{0}^{x} \frac{V_{1}(u)}{u^{3}} d u=\lim _{t \rightarrow+0} I_{2}
$$

whence we get, integrating by parts the left-hand integral and using (3.20) once more,

$$
\lim _{x \rightarrow \infty} \int_{0}^{x} \frac{V(u)}{u^{2}} d u=\lim _{u \rightarrow+0} I_{2} .
$$

The conclusion (3.18) of Theorem B now follows from (3.23) and (3.24).

\section{Applications.}

Theorem 1. Suppose that $\phi(u)$ is subject to conditions C (i)-(iv) of $\S 1$ and $\psi(u)=-\phi^{\prime}(u)$ as there. Suppose that $A(u)$ is a function of bounded variation in every finite interval of $u \geqq 0$ and $A(0)=0$.

If

$$
\begin{gathered}
\liminf _{u \rightarrow \infty} \frac{1}{u} \int_{0}^{u} x d\{A(x)\} \geqq 0, \\
\Phi(t)=\int_{0}^{\infty} \phi(u t) d\{A(u)\}
\end{gathered}
$$

is convergent for $t>0$ and bounded as $t \rightarrow+0$, then

$$
\begin{aligned}
\lim _{u \rightarrow \infty} \inf A(u) & =\underset{u \rightarrow+0}{\liminf } \Phi(t), \\
\limsup _{u \rightarrow \infty} \frac{1}{u} \int_{0}^{u} A(x) d x & =\limsup _{t \rightarrow+0} \Phi(t) .
\end{aligned}
$$


Proof. We can write (4.1) in the form

$$
\begin{aligned}
v(u) & \equiv \frac{1}{u} \int_{0}^{u} x d\{A(x)\} \\
& \equiv A(u)-\frac{1}{u} \int_{0}^{u} A(x) d x=o_{L}(1), \quad u \rightarrow \infty .
\end{aligned}
$$

Then

$$
V(u) \equiv \int_{0}^{u} v(x) d x=\int_{0}^{u}\left\{\sigma_{0}(x)-\sigma_{1}(x)\right\} d x
$$

where

$$
\sigma_{0}(u) \equiv A(u), \quad \sigma_{1}(u) \equiv \frac{1}{u} \int_{0}^{u} A(x) d x
$$

Therefore

$$
\begin{gathered}
\frac{V(u)}{u}=\sigma_{1}(u)-\sigma_{1}^{*}(u), \quad \sigma_{1}^{*}(u) \equiv \frac{1}{u} \int_{0}^{u} \sigma_{1}(x) d x ; \\
t \int_{0}^{\infty} \psi(u t) \frac{V(u)}{u} d u=\Phi_{1}(t)-\Phi_{1}^{*}(t)
\end{gathered}
$$

where

$$
\Phi_{1}(t) \equiv t \int_{0}^{\infty} \psi(u t) \sigma_{1}(u) d u, \quad \Phi_{1}^{*}(t) \equiv t \int_{0}^{\infty} \psi(u t) \sigma_{1}^{*}(u) d u .
$$

By Lemma 2,

$$
\begin{aligned}
\lim _{t \rightarrow+0} \inf \Phi(t) & \leqq \liminf _{t \rightarrow+0} \Phi_{1}(t) \leqq \lim _{t \rightarrow+0} \sup \Phi_{1}^{*}(t) \leqq \limsup _{t \rightarrow+0} \Phi_{1}(t) \\
& \leqq \limsup _{t \rightarrow+0} \Phi(t)
\end{aligned}
$$

and so $\Phi_{1}(t)$ and $\Phi_{1}^{*}(t)$ are bounded as $t \rightarrow+0$ as a result of our assumption in (4.2) that $\Phi(t)$ is bounded as $t \rightarrow+0$. Consequently, from (4.6),

$$
t \int_{0}^{\infty} \psi(u t) \frac{V(u)}{u} d u=O(1), \quad t \rightarrow+0 .
$$

(4.4) and (4.8) show that the hypotheses (3.1), (3.2) of Theorem A obtain with the choice of $v(u)$ in (4.4); while (4.5) shows that, with this choice of $v(u)$, 


$$
\begin{aligned}
\int_{0}^{x} \frac{V(u)}{u^{2}} d u & =\int_{0}^{x} \frac{\sigma_{1}(u)}{u} d u-\int_{0}^{x} \frac{d u}{u^{2}} \int_{0}^{u} \sigma_{1}(\tau) d \tau \\
& =\frac{1}{x} \int_{0}^{x} \sigma_{1}(u) d u \equiv \sigma_{1}^{*}(u)
\end{aligned}
$$

Therefore the conclusion of Theorem $\mathrm{A}$ in the form (3.13) yields

$$
\underset{u \rightarrow \infty}{\operatorname{osc}} \sigma_{1}^{*}(u)=\underset{t \rightarrow+0}{\operatorname{osc}} t \int_{0}^{\infty} \psi(u t) \sigma_{1}^{*}(u) d u \equiv \underset{t \rightarrow+0}{\operatorname{osc}} \Phi_{1}^{*}(t)
$$

Now (4.4) involves

$$
\int_{u}^{u^{\prime}} \frac{v(u)}{u} d u \equiv \sigma_{1}\left(u^{\prime}\right)-\sigma_{1}(u)=o_{L}(1) \log \frac{u^{\prime}}{u}, \quad u^{\prime}>u \rightarrow \infty,
$$

or

$$
\underset{u \rightarrow \infty}{\lim \inf } \underset{u<u^{\prime}<\lambda u}{\text { lower bound }}\left[\sigma_{1}\left(u^{\prime}\right)-\sigma_{1}(u)\right]=o_{L}(1) \log \lambda, \quad u \rightarrow \infty .
$$

Since $\log \lambda \leqq \lambda-1, \lambda>0$, the last step justifies our taking $A(u)$ $=\sigma_{1}(u)$ in Lemma 9 and inferring that

$$
\underset{u \rightarrow \infty}{\operatorname{osc}} \sigma_{1}(u)=\underset{u \rightarrow \infty}{\operatorname{osc}} \sigma_{1}^{*}(u) .
$$

Using (4.10) and (4.7) in (4.9), we obtain

$$
\underset{u \rightarrow \infty}{\operatorname{osc}} \sigma_{1}(u)=\underset{t \rightarrow+0}{\operatorname{osc}} \Phi_{1}^{*}(t) \leqq \underset{t \rightarrow+0}{\operatorname{osc}} \Phi(t) .
$$

But since, from Lemma 2, we have in any case

$$
\underset{u \rightarrow \infty}{\operatorname{osc}} \sigma_{1}(u) \geqq \underset{t \rightarrow+0}{\operatorname{osc}} \Phi(t),
$$

it follows that osc $\sigma_{1}(u)=$ osc $\Phi(t)$, or

(4.11) $\quad \liminf _{u \rightarrow \infty} \sigma_{1}(u)=\liminf _{t \rightarrow+0} \Phi(t), \quad \limsup \sigma_{1}(u)=\limsup _{u \rightarrow+\infty} \Phi(t)$.

In order to pass from (4.11) to our conclusion (4.3) we need only prove that

$$
\liminf _{u \rightarrow \infty} \sigma_{1}(u)=\liminf _{u \rightarrow \infty} \sigma_{0}(u) ;
$$

and this follows from (4.1) by the argument used for the same purpose in the proof of Lemma 9.

Altering our choice of $v(u)$ in $(4.4)$ to $v(u) \equiv u s(u)$, where $s(u)$ is an 
integrable function, but arguing exactly as above, we can establish the following corollary.

COROLLARY 1.1. The general case of Lemma 5 can be restated without the condition lim sup $u s(u)<\infty$, but with the extra condition $F(t)$ $=O(1), t \rightarrow+0$, as well as the differentiability and ultimate monotony of $\psi(u)$ imported from Lemma 4 through Theorem A. form.

In view of Lemmas 9, 7 we can restate Theorem 1 in the following

CoROLlary 1.2. If, in Theorem 1, the hypothesis (4.1) is replaced by $\lim _{u \rightarrow \infty} \inf$ lower bound $\left\{A\left(u^{\prime}\right)-A(u)\right\} \geqq-w(\lambda)=o_{L}(\lambda-1)$ as $1<\lambda \rightarrow 1$

then the conclusion (4.3) can be improved to

$$
\underset{u \rightarrow \infty}{\operatorname{osc}} A(u)=\underset{i \rightarrow+0}{\operatorname{osc}} \Phi(t) .
$$

Corollary 1.2 is substantially Ramaswami's oscillation theorem for the $\Phi$-transform [5, Theorem I.2] while Corollary 1.1 is the similar theorem of Minakshisundaram [2, Theorem 3].

Theorem 2. Let $\phi(u)$ fulfill the conditions $\mathrm{C}(\mathrm{i})-(\mathrm{iv})$ of $\$ 1$ and further let $\psi(u)=-\phi^{\prime}(u)$ be such that

$$
\int_{0}^{\infty} u^{i x} \psi(u) d u \neq 0 \text { for any real } x \text {. }
$$

If

$$
\begin{gathered}
\liminf _{u \rightarrow \infty} \frac{1}{u} \int_{0}^{u} x d\{A(x)\}>-\infty, \\
\Phi(t)=\int_{0}^{\infty} \phi(u t) d\{A(u)\}
\end{gathered}
$$

exists for $t>0$ and converges as $t \rightarrow+0$, then

$$
\lim _{u \rightarrow \infty} \frac{A_{1}(u)}{u}=\lim _{t \rightarrow+0} \Phi(t), \quad A_{1}(u) \equiv \int_{0}^{u} A(x) d x .
$$

Proof. We can state (4.12) in the form

$$
\frac{V(u)}{u} \equiv \frac{1}{u} \int_{0}^{u} x d\{A(x)\} \equiv A(u)-\frac{A_{1}(u)}{u}=O_{L}(1), \quad u \rightarrow \infty .
$$


Then

$$
\begin{array}{rlr}
t \int_{0}^{\infty} \psi(u t) \frac{V(u)}{u} d u & =\Phi(t)-\Phi_{1}(t) & \\
= & o(1), & t \rightarrow+0 \\
\Phi_{1}(t) \equiv t \int_{0}^{\infty} \psi(u t) \frac{A_{1}(u)}{u} d u, & t>0
\end{array}
$$

since, by hypothesis, $\Phi(t) \rightarrow A$ (say) as $t \rightarrow+0$ and, by Lemma $2, \Phi_{1}(t)$ $\rightarrow A$ as $t \rightarrow+0$. (4.15) and (4.16) are the same as the hypotheses (3.15) and (3.16) of Theorem B, with our choice of $V(u)$. Therefore, the conclusion (3.18) of Theorem B holds with

$$
\int_{0}^{x} \frac{V(u)}{u^{2}} d u=\int_{0}^{x} \frac{A(u)}{u} d u-\int_{0}^{x} \frac{A_{1}(u)}{u^{2}} d u=\frac{A_{1}(x)}{x}
$$

and gives us

$$
\lim _{x \rightarrow \infty} \frac{A_{1}(x)}{x}=\lim _{t \rightarrow+0} t \int_{0}^{\infty} \psi(u t) \frac{A_{1}(u)}{u} d u \equiv \lim _{t \rightarrow+0} \Phi_{1}(t)
$$

which leads to the desired conclusion on account of (4.16).

Taking into account Lemma 7, we get at once from Theorem 2 the following corollary.

COROLLARY 2.1. Theorem 2 can be restated with (4.12) changed to

$$
\underset{u<u^{\prime}<\lambda u}{\operatorname{lower}}\left\{A\left(u^{\prime}\right)-A(u)\right\} \geqq-w(\lambda) \text { for every } u>0, \lambda>1
$$

but without any other change.

Combining Corollary 2.1 with Lemma 8 , we are obviously led to the following corollary.

COROLLARY 2.2. Theorem 2 can be restated with the hypothesis (4.12) changed to

$\underset{u \rightarrow \infty}{\lim \inf } \underset{u<u^{\prime}<\lambda u}{\text { lower bound }}\left\{A\left(u^{\prime}\right)-A(u)\right\} \geqq-w(\lambda) \rightarrow 0$ as $1<\lambda \rightarrow 1$,

and the conclusion (4.14) changed to

$$
\lim _{u \rightarrow \infty} A(u)=\lim _{t \rightarrow+0} \Phi(t) .
$$

Corollary 2.1 is essentially a combination of two theorems of Karamata [1, Sätzen A, B] while Corollary 2.2 is a combination of three of his theorems [1, Sätzen A, B, C]. 
REMARKS. (i) The following are well known examples of kernels which satisfy the conditions $C(i)-(i v)$ and the additional condition

$$
\begin{array}{ll}
\int_{0}^{\infty} u^{i x} \psi(u) d u \neq 0 & (-\infty<x<\infty) . \\
\phi(u)=e^{-u}, u \geqq 0 & \text { [Laplace kernel]; } \\
\phi(u)=(1+u)^{-\rho}, \rho>0, u \geqq 0 & \text { [Stieltjes kernel]; } \\
\phi(u)=u /\left(e^{u}-1\right), u>0 & \text { [Lambert kernel]. }
\end{array}
$$

Theorems A and B, as well as Theorems 1 and 2 following from them, are true for each of these kernels.

(ii) All the familiar applications of Theorem 2 to Dirichlet's series [7, Theorems 3-6] can be carried out in like manner for the series

$$
\Phi(t)=\sum_{n=1}^{\infty} a_{n} \phi\left(\lambda_{n} t\right), \quad t>0,0<\lambda_{1}<\lambda_{2}<\cdots, \lambda_{n} \rightarrow \infty,
$$

obtained from (4.13), taking for $A(u)$ the $\lambda$-step function:

$$
\begin{aligned}
A(u)= & a_{1}+a_{2}+\cdots+a_{n} & \text { for } \lambda_{n} \leqq u<\lambda_{n+1}, \\
& A(u)=0 & \text { for } 0 \leqq u<\lambda_{1},
\end{aligned}
$$

$\phi(u)$ being either the Stieltjes kernel or the Lambert kernel.

(iii) Corollaries 2.1 and 2.2 are limiting cases of results [4, Theorem 2] in which the hypothesis on

$$
\underset{u<u^{\prime}<\lambda u}{\text { lower bound }}\left\{A\left(u^{\prime}\right)-A(u)\right\}
$$

of either corollary is changed to a similar hypothesis on

$$
\underset{\substack{u<u^{\prime}<\lambda u \\ \text { lower bound }}}{ }\left\{A\left(u^{\prime}\right)-A(u)\right\} / u^{\beta}, \quad \beta>0 .
$$

\section{REFERENCES}

1. J. Karamata, Über die O-Inversionssätze der Limitierungsverfahren, Math. Zeit. vol. 37 (1933) pp. 582-588.

2. S. Minakshisundaram, On generalised Tauberian theorems, Math. Zeit. vol. 45 (1939) pp. 495-506.

3. C. T. Rajagopal, $A$ note on the oscillation of Riesz means of any order, J. London Math. Soc. vol. 21 (1946) pp. 275-282.

4. - A note on 'positive' Tauberian theorems, J. London Math. Soc. vol. 25 (1950) pp. 315-327.

5. V. Ramaswami, The generalized Abel-Tauber theorem, Proc. London Math. Soc. (2) vol. 41 (1936) pp. 408-417.

6. O. Szász, Über einige Sätze von Hardy und Littlewood, Nachr. Ges. Wiss. Göttingen (1930) pp. 315-333. 
7. - Converse theorems of summability for Dirichlet's series, Trans. Amer. Math. Soc. vol. 39 (1936) pp. 117-130. 1946.

8. D. V. Widder, The Laplace transform, Princeton Mathematical Series, no. 6 ,

Madras Christian College

\section{TYPICALLY-REAL FUNCTIONS WITH ASSIGNED ZEROS}

\section{A. W. GOODMAN}

1. Introduction. A function $f(z)$

$$
f(z)=\sum_{n=0}^{\infty} b_{n} z^{n}
$$

is said to be typically-real of order $p$, if in (1.1) the coefficients $b_{n}$ are all real and if either (I) $f(z)$ is regular in $|z| \leqq 1$ and $\Im f\left(e^{i \theta}\right)$ changes sign $2 p$ times as $z=e^{i \theta}$ traverses the boundary of the unit circle, or (II) $f(z)$ is regular in $|z|<1$ and if there is a $\rho<1$ such that for each $r$ in $\rho<r<1, \Im f\left(r e^{i \theta}\right)$ changes sign $2 p$ times as $z=r e^{i \theta}$ traverses the circle $|z|=r$. This set of functions is denoted by $T(p)$.

The name typically-real was first suggested by Rogosinski [6] ${ }^{1}$ who studied these functions in the case $p=1$. The more general set of functions $T(p)$ was first introduced by Robertson $[5 ; 4]$, and in a recent paper by Robertson and Goodman [3] the sharp upper bound for $\left|b_{n}\right|$ in terms of $\left|b_{1}\right|, \cdots,\left|b_{p}\right|$ was obtained, namely for $n=p$ $+1, p+2, \cdots$,

$$
\left|b_{n}\right| \leqq \sum_{k=1}^{p} \frac{2 k(n+p) !}{(p+k) !(p-k) !(n-p-1) !\left(n^{2}-k^{2}\right)}\left|b_{k}\right| .
$$

We shall see in what follows that the size of $\left|b_{n}\right|$ is also governed by the locations of the zeros of $f(z)$ for functions of the set $T(p)$. More precisely we shall prove the following theorem.

THEOREM 1. Let

$$
f(z)=z^{q}+\sum_{n=q+1}^{\infty} b_{n} z^{n}
$$

Presented to the Society, December 29, 1950; received by the editors May 19, 1950 and, in revised form, June 15, 1950.

${ }_{1}$ Numbers in brackets refer to the references at the end of the paper. 